\title{
Pure Lymphoepithelioma-Like Carcinoma Originating from the Urinary Bladder
}

\author{
Takashi Nagai $^{a}$ Taku Naiki ${ }^{a}$ Noriyasu Kawai ${ }^{a} \quad K^{2}$ itaro Iida ${ }^{a}$ \\ Toshiki Etani $^{\mathrm{a}}$ Ryosuke Ando ${ }^{\mathrm{a}}$ Shuzo Hamamoto ${ }^{\mathrm{a}}$ Yosuke Sugiyama $^{\mathrm{b}}$ \\ Atsushi Okada $^{a}$ Kentaro Mizuno ${ }^{a}$ Yukihiro Umemoto ${ }^{a}$ Takahiro Yasui ${ }^{a}$ \\ ${ }^{a}$ Department of Nephro-Urology, Graduate School of Medical Sciences, Nagoya City \\ University, and ${ }^{\mathrm{b}}$ Department of Pharmacy, Nagoya City University Hospital, Nagoya, Japan
}

\section{Key Words}

Lymphoepithelioma-like carcinoma - Urinary bladder - Epstein-Barr virus · Apparent diffusion coefficient value

\begin{abstract}
Lymphoepithelioma-like carcinoma of the urinary bladder (LELCB) is a rare variant of infiltrating urothelial carcinoma. We report a case of LELCB in a 43-year-old man. Ultrasonography and cystoscopy revealed two bladder tumors, one on the left side of the trigone and the other on the right side of the trigone. Transurethral resection of the bladder tumors was performed and pathological analysis revealed undifferentiated carcinoma. We therefore performed radical cystectomy and urinary diversion. Immunohistochemically the tumor cells were positive for cytokeratin, but negative for Epstein-Barr virus-encoded small RNA in situ hybridization as found for previous cases of LELCB. The final pathological diagnosis was a lymphoepithelioma-like variant of urothelial carcinoma with perivesical soft tissue invasion. For adjuvant systemic chemotherapy, three courses of cisplatin were administered. The patient subsequently became free of cancer 72 months postoperatively. Based on the literature, pure or predominant LELCB types show favorable prognoses due to their sensitivity to chemotherapy or radiotherapy. An analysis of the apparent diffusion coefficient (ADC) values of bladder tumors examined in our institution revealed that the ADC value measured for this LELCB was relatively low compared to conventional urothelial carcinomas. This suggests that measuring the $A D C$ value of a lymphoepithelioma-like carcinoma prior to operation may be helpful in predicting LELCB.

\section{KARGER}

Taku Naiki

Department of Nephro-Urology, Graduate School of Medical Sciences

Nagoya City University, Kawasumi 1, Mizuho-cho, Mizuho-ku

Nagoya 467-8601 (Japan)

E-Mail naiki@med.nagoya-cu.ac.jp or rx-nike@hotmail.co.jp 
Nagai et al.: Pure Lymphoepithelioma-Like Carcinoma Originating from the Urinary Bladder

\section{Introduction}

Lymphoepithelioma is an undifferentiated carcinoma of the nasopharynx. In comparison, a lymphoepithelioma-like carcinoma (LELC) is a tumor which mimics lymphoepithelioma in terms of its pathological features. The primary lesion of LELC occurs in the stomach, lungs, thymus, salivary glands or urinary bladder [1]. LELC of the urinary bladder (LELCB) is a rare variant of infiltrating urothelial carcinoma and was first described by Zukerberg et al. in 1991 [2]. Including this case, there are merely 105 cases in the English language medical literature, with only 8 cases reported as originating from Asian countries [3-6]. In 1994, Amin et al. [7] defined three types of LELCB based on the amount of LELC in the tumor pure $(100 \%)$, predominant $(50-99 \%)$ and focal $(<50 \%)-$, with the three types determining a patient's prognosis. We herein report a case of LELCB, with a preoperative diagnosis of an undifferentiated carcinoma, in which radical cystectomy and systemic chemotherapy were successfully undertaken. Based on previous case reports of LELCB in the English language medical literature, we examined the prognosis for LELCB. In addition, we analyzed the preoperative diagnostic characteristics of this LELCB by measuring its apparent diffusion coefficient (ADC) value by magnetic resonance imaging (MRI) and compared this to those of past cases of urothelial carcinoma of the urinary bladder at our institution.

\section{Case Report}

A 43-year-old Japanese man presented to our department complaining of frequent urination. He had no prior personal or family medical history of this complaint. There were no notable findings in blood tests and chest radiography. Urine cytology results were negative. On cystoscopy, two bladder tumors were observed, one on the left side of the trigone and the other on the right side of the trigone. A total body computed tomography (CT) scan was performed and the presence of distant metastases or lymph node involvement was excluded. MRI revealed that the tumor on the left side of the trigone had invaded the perivesical soft tissue of the bladder, but not the prostate or seminal vesicles (fig. 1a, b).

A transurethral resection of bladder tumor (TURBT) was performed. Pathological findings revealed undifferentiated atypical epithelial cells with enlarged nuclei that had formed into sheets to invade the muscle layer. These results indicated a muscle-invasive pT2 undifferentiated carcinoma of the urinary bladder. Radical cystectomy and ileal conduit diversion were performed. Pathological findings revealed large, undifferentiated carcinoma cells with pleomorphic nuclei and prominent nucleoli, the infiltration of inflammatory cells consisting predominantly of lymphocytes, and the reach of their invasion to the perivesical soft tissue of the bladder (fig. 2a, b). The final pathological diagnosis was an invasive urothelial carcinoma, lymphoepithelioma-like variant, pT3. Tumor cells were positive for cytokeratin (AE1/ AE3) (fig. 2c) and E-cadherin (fig. 2d). As adjuvant chemotherapy, cisplatin-based systemic chemotherapy was performed, and cancer recurrence was not apparent at the usual followup as determined by CT scan and cytological analysis. The patient remained alive and free of cancer 72 months postoperatively.

\section{Discussion}

According to the WHO classification criteria, LELCB is defined as a subtype of undifferentiated carcinomas. To date 105 cases, including this one, have been described in the En- 
Nagai et al.: Pure Lymphoepithelioma-Like Carcinoma Originating from the Urinary Bladder

glish language medical literature. LELCB shows carcinomatous components contrasting with lymphocyte infiltration and mimics chronic inflammation or malignant lymphoma; immunohistochemical stains such as for cytokeratin are helpful in distinguishing between these diseases [6, 7]. Between 1991 and June 2015, 84 cases of LELCB, according to the classification of Amin et al. [7], were reported in the English language medical literature. We allocated the 84 cases to two groups - pure/predominant and focal - and investigated their prognoses based on previous reports. Seventy patients (83\%) formed the pure/predominant group and 14 patients (17\%) formed the focal group. Kaplan-Meier curves of overall survival rates of the pure/predominant and focal groups are shown in figure 3. As a result, the pure/predominant group had as significantly better prognosis than the focal type group (log-rank test, $\mathrm{p}<0.001$ ) (fig. 3). The predominant or focal types of LELCB include squamous cell carcinoma, adenocarcinoma and urothelial carcinoma. Containing only LELC, this case seemed to have the characteristics of a pure type of LELCB. Unfortunately, there is at present no established treatment for LELCB because of its rarity as a bladder tumor subtype, with few reports existing for this disease. Some reports describe LELCB as being sensitive to cisplatin, while others suggest that it is possible to preserve the bladder in the case of a pure or predominant type, even if the tumor has invaded the muscle $[4,8-10]$. We conducted a radical cystectomy because TURBT revealed an undifferentiated carcinoma pT2. Adjuvant cisplatin-based chemotherapy was also instituted because radical cystectomy yielded a diagnosis of LELCB pT3. The combination of external beam radiotherapy and chemotherapy to preserve the bladder may be considered a suitable treatment option if the diagnosis prior to radical cystectomy is accurate.

Epstein-Barr virus (EBV) is associated with lymphoepithelioma or LELC of several tissues (lung, stomach and salivary glands), except the bladder. A close reading of the literature suggests that if tumor cells are positive for EBV RNA, the tumor might be a metastasis from another tissue [1]. Therefore, we performed EBV-encoded small RNA in situ hybridization (EBER-ISH), but this did not reveal a relationship between EBV and LELCB in this case (fig. 2e). However, in treating LELCB, EBER-ISH is useful and necessary to rule out the tumor as being a metastasis from another tissue.

MRI is widely used in local staging or to detect lymph node metastases, and is essential for determining suitable treatments for bladder tumors. Recently, measuring ADC values has been suggested to be helpful in diagnosing bladder tumors [11]. To further improve preoperative diagnostic accuracy, we retrospectively measured the ADC value of this case. The mean value was found to be $782 \times 10^{-3} \mathrm{~mm}^{2} / \mathrm{s}$ (fig. 1c), which was relatively lower than the mean values we found for muscle-invading urothelial carcinomas studied at our institution (56 cases, median $1,047 \times 10^{-3} \mathrm{~mm}^{2} / \mathrm{s}$; see online supplementary material, www.karger.com/doi/10.1159/000445049). In urothelial carcinoma, in 2014 Yamada et al. [12] reported a correlation between high ADC values and good histopathological features of bladder tumors which is the case for urothelial carcinoma. However, in spite of the relatively low ADC value, the prognosis of this case was relatively good as previously described. The low ADC value calculated in this case may be due to the fact that the observed lymphocyte infiltration probably caused an increase in cell density and a decrease in diffusion capacity. There are several pitfalls and limitations in detecting pathological characteristics of tumors [11], and further consideration will have to be given to the usefulness of measuring ADC values of bladder tumors. Regardless, though LELCB is a rare tumor, measuring ADC values may be useful in predicting the histopathological characteristics of such bladder tumors. 


\section{Case Reports in Oncology}

\begin{tabular}{l|l}
\hline Case Rep Oncol 2016;9:188-194 \\
\hline DOI: 10.1159/000445049 & $\begin{array}{l}\text { ○ 2016 The Author(s). Published by S. Karger AG, Basel } \\
\text { www.karger.com/cro }\end{array}$ \\
\hline
\end{tabular}

Nagai et al.: Pure Lymphoepithelioma-Like Carcinoma Originating from the Urinary Bladder

\section{Conclusion}

We treated a rare case of LELCB, classed as a pure type and with a better prognosis than the focal type according to the classification of Amin et al. [7]. It is necessary to consider a diagnosis of LELCB when TURBT indicates an undifferentiated carcinoma. Additionally, measuring the ADC value of a urinary bladder tumor may be helpful in predicting its pathological features.

\section{Statement of Ethics}

Written informed consent was obtained from the patient for publication of this case report and accompanying images. A copy of the written consent is available for review from the Editor-in-Chief of this journal. This study was conducted according to the Declaration of Helsinki.

\section{Disclosure Statement}

There are no potential conflicts of interest. The authors received no financial support for the research, authorship and/or publication of this paper.

\section{References}

1 Porcaro AB, Gilioli E, Migliorini F, Antoniolli SZ, Iannucci A, Comunale L: Primary lymphoepithelioma-like carcinoma of the urinary bladder: report of one case with review and update of the literature after a pooled analysis of 43 patients. Int Urol Nephrol 2003;35:99-106.

2 Zukerberg LR, Harris NL, Young RH: Carcinomas of the urinary bladder simulating malignant lymphoma. A report of five cases. Am J Surg Pathol 1991;15:569-576.

-3 Pan ST, Wang RC, Liu MY, Chuang SS: Lymphoepithelioma-like carcinoma of the urinary bladder: a report of two cases. Anal Quant Cytopathol Histpathol 2013;35:344-348.

4 Yoshino T, Ohara S, Moriyama H: Lymphoepithelioma-like carcinoma of the urinary bladder: a case report and review of the literature. BMC Res Notes 2014;7:779.

5 Fujino T, Kubota M, Nishiyama R, Kanno T, Okada T, Higashi Y, Yamada H, Okamoto E: A case of lymphoepithelioma-like carcinoma of the bladder (in Japanese). Hinyokika Kiyo 2014;60:507-511.

-6 Kushida N, Kushakabe T, Kataoka M, Kumagai S, Aikawa K, Kojima Y: External beam radiotherapy for focal lymphoepithelioma-like carcinoma in the urinary bladder: a case report and literature review. Case Rep Oncol 2015;8:15-20.

7 Amin MB, Ro JY, Lee KM, Ordóñez NG, Dinney CP, Gulley ML, Ayala AG: Lymphoepithelioma-like carcinoma of the urinary bladder. Am J Surg Pathol 1994;18:466-473.

-8 Tamas EF, Nielsen ME, Schoenberg MP, Epstein JI: Lymphoepithelioma-like carcinoma of the urinary tract: a clinicopathological study of 30 pure and mixed cases. Mod Pathol 2007;20:828-834.

-9 Mayer EK, Beckley I, Winkler MH: Lymphoepithelioma-like carcinoma of the urinary bladder - diagnostic and clinical implications. Nat Clin Pract Urol 2007;4:167-171.

10 Kozyrakis D, Petraki C, Prombonas I, Grigorakis A, Kanellis G, Malovrouvas D: Lymphoepithelioma-like bladder cancer: clinicopathologic study of six cases. Int J Urol 2011;18:731-734.

-11 Lin WC, Chen JH: Pitfalls and limitations of diffusion-weighted magnetic resonance imaging in the diagnosis of urinary bladder cancer. Transl Oncol 2015;8:217-230.

12 Yamada Y, Kobayashi S, Isoshima S, Arima K, Sakuma H, Sugimura Y: The usefulness of diffusion-weighted magnetic resonance imaging in bladder cancer staging and functional analysis. J Cancer Res Ther 2014;10 878-882.

13 Kanda Y: Investigation of the freely available easy-to-use software 'EZR' for medical statistics. Bone Marrow Transplant 2013;48:452-458. 


\section{Case Reports in Oncology}

\begin{tabular}{l|l}
\hline Case Rep Oncol 2016;9:188-194 \\
\hline DOI: 10.1159/000445049 & $\begin{array}{l}\text { ○ 2016 The Author(s). Published by S. Karger AG, Basel } \\
\text { www.karger.com/cro }\end{array}$ \\
\hline
\end{tabular}

Nagai et al.: Pure Lymphoepithelioma-Like Carcinoma Originating from the Urinary Bladder
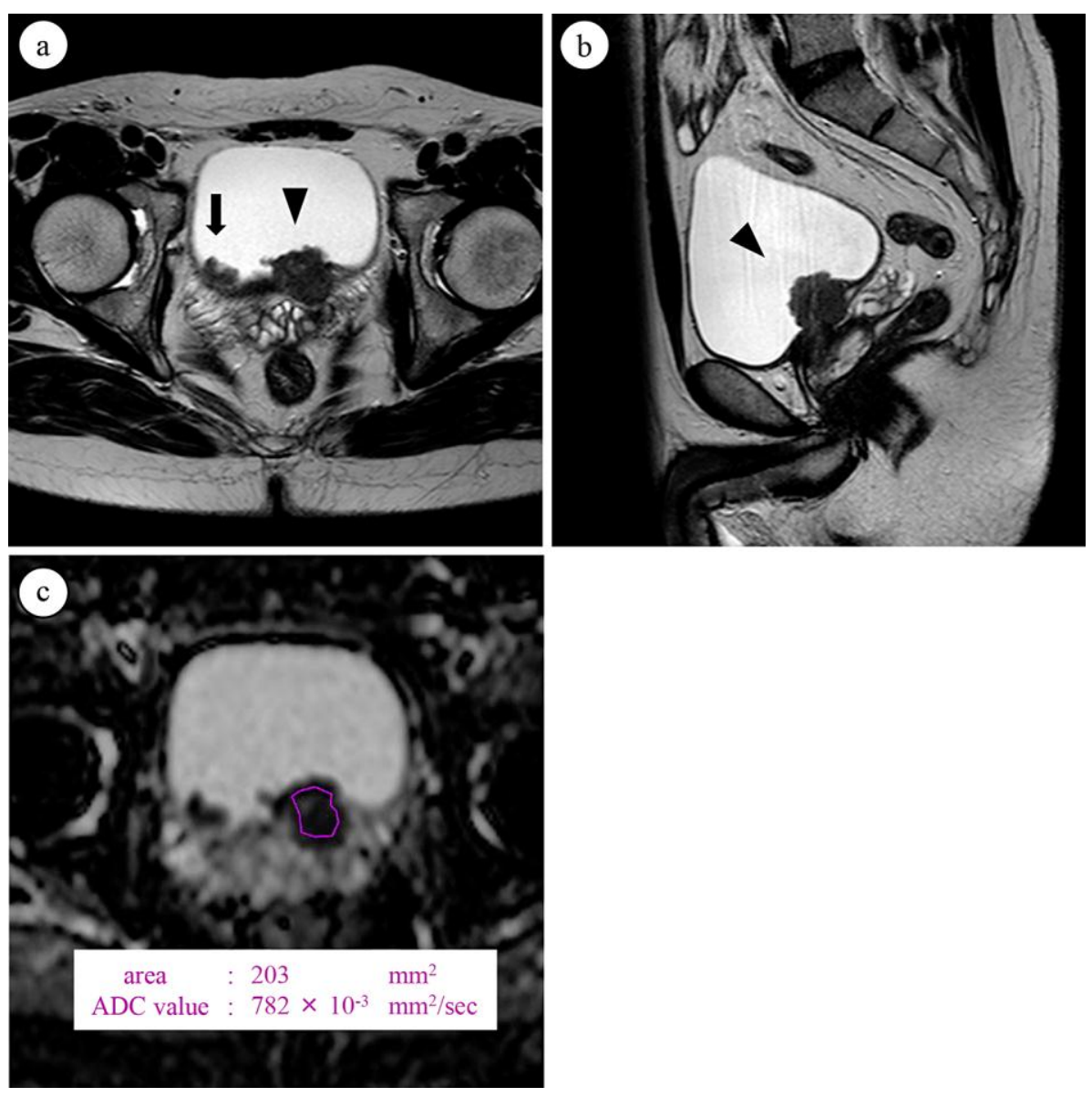

Fig. 1. a MRI (T2-enhanced, coronal image) showing two bladder tumors, one on the left side of trigone (arrowhead) and the other on the right side of the trigone (arrow). b MRI (T2-enhanced, sagittal image) showing a bladder tumor on the left side of the trigone (arrowhead) and perivesical soft tissue invasion. c The average value of the ADC of the tumor was $782 \times 10^{-3} \mathrm{~mm}^{2} / \mathrm{s}$. 


\section{Case Reports in Oncology}

\begin{tabular}{l|l}
\hline Case Rep Oncol 2016;9:188-194 \\
\hline DOI: 10.1159/000445049 & $\begin{array}{l}\text { C 2016 The Author(s). Published by S. Karger AG, Basel } \\
\text { www.karger.com/cro }\end{array}$ \\
\hline
\end{tabular}

Nagai et al.: Pure Lymphoepithelioma-Like Carcinoma Originating from the Urinary Bladder
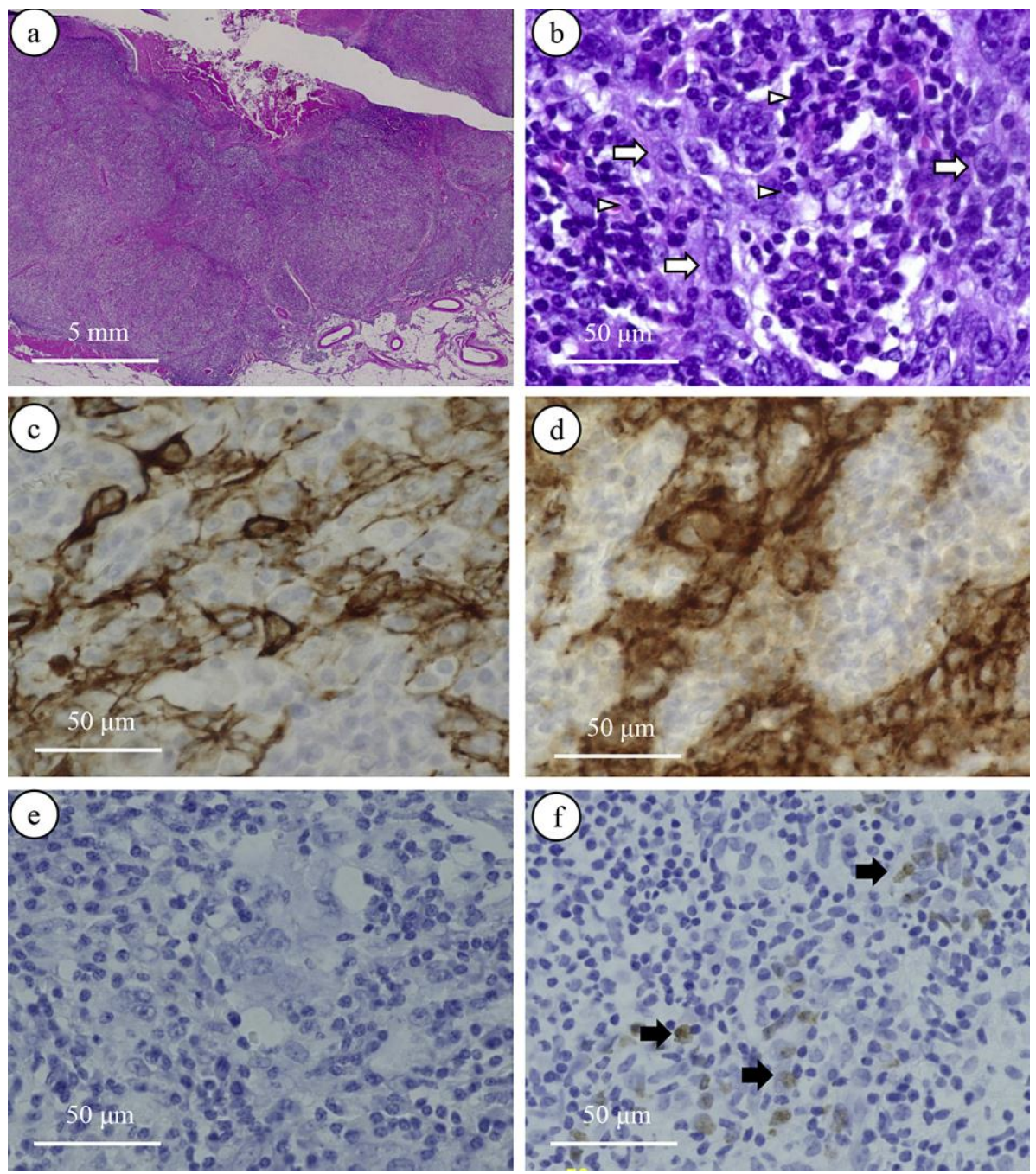

Fig. 2. a-d LELCB in this case. a Microscopic findings at low magnification showed that the tumors had invaded the perivesical soft tissue. H\&E stain. b Microscopic findings at high magnification showed that tumor cells (arrows) were surrounded by infiltrating lymphocytes (arrowheads). H\&E stain. c Immunohistochemical staining for cytokeratin (AE1/AE3). d Immunohistochemical staining for E-cadherin. e EBER-ISH for this case. Tumor cells were negative. $f$ Lymphoepithelioma of the pharynx in another patient. EBER-ISH of tissues. Tumor cells (arrows) were positive for EBER-ISH (positive control). 


\section{Case Reports in \\ Oncology}

\begin{tabular}{l|l|}
\hline Case Rep Oncol 2016;9:188-194 \\
\hline DOI: 10.1159/000445049 & $\begin{array}{l}\text { @ 2016 The Author(s). Published by S. Karger AG, Basel } \\
\text { www.karger.com/cro }\end{array}$ \\
\hline
\end{tabular}

Nagai et al.: Pure Lymphoepithelioma-Like Carcinoma Originating from the Urinary Bladder

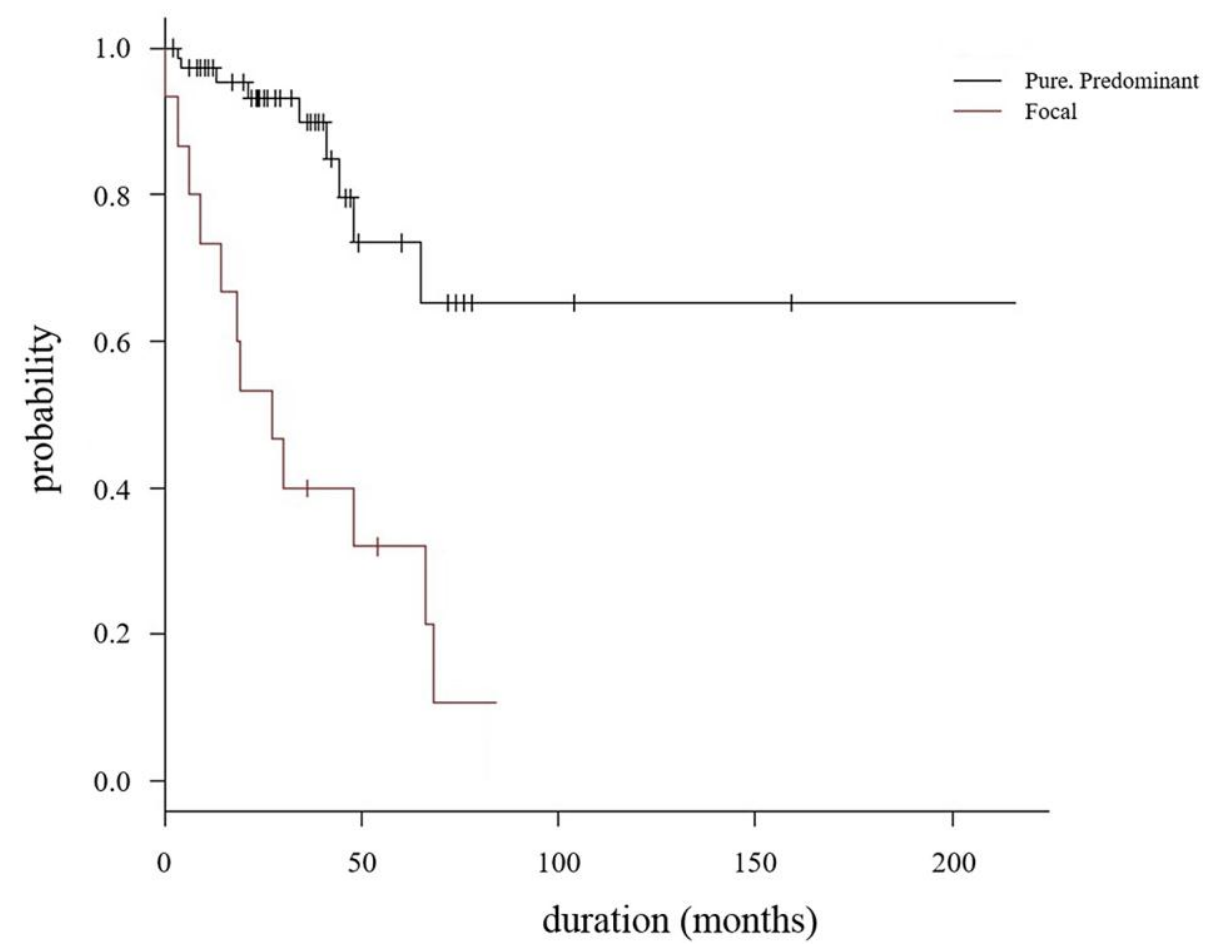

Fig. 3. Kaplan-Meier curves of overall survival rates for pure/predominant and focal types of LELCB. 